\title{
OBSERVATÓRIO DE INOVAÇÃO COMO FERRAMENTA DE SUBSÍDIO À INTELIGÊNCIA NAS ORGANIZAÇÕES
}

Thiago Zschornack ${ }^{1}$ João Artur de Souza ${ }^{2}$ Luana Siewert Pretto ${ }^{3}$ Adriana Falcão Loth ${ }^{4}$

\section{RESUMO}

Com cenários sociais, políticos e econômicos cada vez mais dinâmicos,importantes transformações vêm acontecendo em nossa sociedade, levando as empresas a enfrentarem os mais variados e complexos problemas. Assim, ideias inovadoras são fundamentais para dar resposta às demandas do mercado e para a resolução dos principais problemas organizacionais. $\mathrm{O}$ processo de inovação não ocorre na empresa de forma isolada, ele é interativo e de natureza social, contando com a contribuição de vários agentes econômicos e sociais, detentores de diferentes tipos de informação e conhecimento, dentro e fora da empresa. O Observatório de Inovação é um espaço para mostrar, articular, apoiar e acompanhar os atores que compõem a rede do Ecossistema de Inovação nas organizações, atuando, assim, como um mecanismo de inteligência que apoia o processo de inovação empresarial. Desta forma, o presente artigo tem como objetivo apresentar os principais conceitos e modelos de Observatórios de Inovação existentes na literatura, bem como destacar a sua importância como elemento de subsídio para a tomada de decisão em processos que envolvam inovação. A metodologia utilizada foi a pesquisa bibliográfica, a qual se deu basicamente em fontes secundárias de dados (base SCOPUS e SCIELO), correlacionando os temas gestão do conhecimento, inovação, inteligência e observatórios. Conclui-se que existem várias iniciativas no Brasil e no mundo, porém nota-se a falta de um padrão quanto aos objetivos pretendidos, escopo de abrangência e resultados obtidos. Ou seja, o maior desafio reside em tornar cada vez mais aplicáveis as informações organizadas e disseminadas pelos Observatórios.

Palavras-Chave: Gestão do conhecimento. Inovação. Observatório de Inovação. 


\section{INTRODUÇÃO}

Dentre os diversos tipos de informações geradas pela sociedade, a informação científica e tecnológica se destaca pela sua respeitável função no desenvolvimento de um país. A informação gerada pela ciência e transformada em tecnologia é essencial para a competitividade em função da inovação que pode alavancar (CASTRO; JANNUZZI; MATTOS, 2007 apud RONCAGLIO, 2008).

A inovação pode ser entendida como o uso do conhecimento e das competências organizacionais com o objetivo de criar valor, sendo aplicada nos processos para melhorar a qualidade dos bens/serviços, reduzir tempos e custos, entre outros objetivos organizacionais (VARVAKIS et al.,2010).Em termos mais amplos, a inovação é “[...] a exploração de novas ideias para melhorar os negócios, criando vantagens competitivas e gerando sucesso no mercado.”(OECD,2005,p. 13). Além disso, [...] é "movida pela habilidade de estabelecer relações, detectar oportunidades e tirar proveito das mesmas"(TIDD; BESSANT; PAVITT,2008,p.23), seja pela abertura de novos mercados, ou servindo de formas diferentes os mercados já existentes.

Considerando que o ato de inovar é geralmente uma decisão que perpassa a alta gestão, é fundamental que existam informações completas e precisas que subsidiem uma tomada de decisão inteligente. Ou seja, a inovação precisa ser uma decisão pautada em informações e premissas que garantam vantagens para a organização, tais como: vantagem competitiva, redução de custos, melhoria na satisfação dos clientes etc.

Nesse sentido, uma ferramenta que tem se mostrado bastante útil para subsidiar boas decisões de inovação é o Observatório de Inovação. O Observatório é um espaço para mostrar, articular, apoiar e acompanhar os atores que compõem a rede do Ecossistema de Inovação de uma entidade, sejam eles atores de suporte ou iniciativas de inovação. Assim, o Observatório de Inovação produz e sistematiza informações estratégicas com ênfase em tecnologias, tendências de mercado e inovação, voltadas às necessidades da empresa e à sua acumulação de capacidade de inovação (OBISF, 2019).

Este artigo tem por objetivo apresentar os principais conceitos e modelos de Observatórios de Inovação presentes na literatura (base SCOPUS e SCIELO), bem como o seu papel no processo de tomada de decisão quando o assunto envolver inovação. Desta forma, este artigo está estruturado em duas partes principais. A primeira parte foca na definição dos principais conceitos relacionados a conhecimento, inovação e observatórios. E a segunda parte destaca os tipos, papeis e a importância dos Observatórios de Inovação no processo de inovação empresarial. 
Nesta seção, será apresentada a fundamentação teórica que deu subsídio para a construção do estudo, a qual foi estruturada a partir dos conceitos de inovação, conhecimento e observatórios.

\subsection{Inovação e conhecimento}

Segundo Miguez (2017), a inovação não é tema novo, contudo, foi em período recente que tal discussão ganhou popularidade midiática, tanto em discursos do governo, empresas e universidades.

Inovação pode ser entendida como um processo pelo qual as novas ideias se tornam realidade (BARBIERI; ÁLVARES, 2005), resultando em melhorias, ganhos ou lucros para a empresa (GUNDLING;PORRAS,2000), representando uma importante fonte de vantagem competitiva (ČERNE;JAKLIČ; ŠKERLAVAJ,2013) e, para sustentar uma vantagem competitiva em um ambiente global, a inovação contínua é crucial (DAMANPOUR; WALKER; AVELLANEDA, 2009).

O processo de inovação inclui várias etapas, sendo a primeira e, talvez, mais importante, a criatividade, formalmente definida como a geração de ideias novas e úteis, sendo o ponto óbvio de partida para que a inovação ocorra (ŠKERLAVAJ; ČERNE; DYSVIK, 2014).
A capacidade de uma empresa para crescer depende de seu potencial de gerar novas ideias e explorá-las de forma eficaz para o seu benefício emlongo prazo(FLYNN etal.,2003).Assim, as empresas devem gerar um número e variedade suficiente de ideias de alta qualidade para obter um bem equilibrado portfólio de projetos de inovação com potencial de sucesso (KOCK; HEISING; GEMUENDEN, 2015), pois dependem cada vez mais de desenvolvimento de novos produtos, de equipes para gerar ideias criativas e moldar essas ideias inovadoras em novos produtos ou serviços (LIU; CHEN;TAO, 2015 apud CASTRO, 2016).

Sob a perspectiva do conhecimento inovação, é o processo de converter conhecimento em valor, pela implementação de novos ou melhores produtos, processos e sistemas (FERRARESI et al., 2012). Inovação é a criação de novos conhecimentos e ideias, para facilitar novos resultados de negócios, visando à melhoria dos processos internos e estruturas, criando produtos e serviços adaptados ao mercado (DU PLESSIS,2007) e que atendam às necessidades e aos desejos do consumidor (FIEDLER,2011). A gestão da inovação envolve a gestão de conhecimentos em um contexto claro e definido (MOUSTAGHFIR et al.,2013). Sendo assim, um dos componentes centrais da inovação é o conhecimento(RUIZ-JIMÉNEZ;DELMAR FUENTES-FUENTES, 2013). 
A inovação pode ser criada por meio do conhecimento que pode existir através de experiências vividas, ou por processo de busca, de mercado, por tecnologia, ações da concorrência etc. Ao combinar os diferentes tipos de conhecimento, ocorrem condições de alta incerteza em uma inovação bem-sucedida, que será diminuída com a capacidade de transformar essas incertezas em conhecimento com a prática de uma ação de equilíbrio, utilizando recursos para reduzir as incertezas, ou seja, isso é gestão da inovação (TIDD; BESSANT; PAVITT,2008).

Figura 1: Relação Conhecimento x Inovação

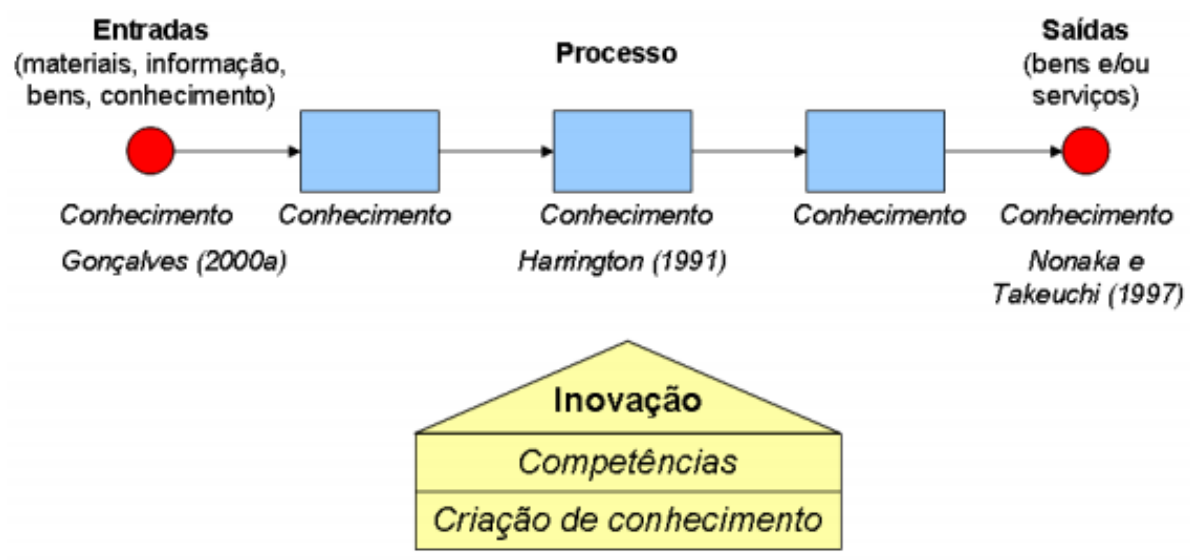

Fonte: Nonaka e Takeuchi (1997)

A Gestão do Conhecimento (GC), como área de estudo,surgiu em movimentos internacionais nos finais dos anos de 1990 impulsionadas por pesquisadores e trabalhadores do conhecimento, como Davenport e Prusak (1998) e Nonaka e Takeuchi (1997). Em uma organização, a GC está relacionada à tomada de decisões estratégicas que a impactam como um todo e indicam processos de gestão contribuindo para a melhoria na qualidade de produtos e serviços, na produtividade, na rentabilidade e no crescimento. Neste sentido, a GC pode ser entendida, segundo Sveiby (1998), como “[...] a arte de gerar valor a partir de bens intangíveis da organização [...]”, ou seja, sua função é gerar riqueza e valor a partir do gerenciamento de elementos que estão fora do contexto habitual de terra, capital e mão de obra, visão esta compartilhada por Drucker (1994). Logo, percebe-se que, para ter um diferencial competitivo, as organizações do século XXI, nas quais o conhecimento é artefato de valor, precisam estar atentas à inovação, que pode ser expressa por meio de um novo produto, processo ou sistema com potencial de geração de valor competitivo no mercado.

\subsection{Observatórios}

\subsubsection{Definição e contextualização}

Observatório é um sistema organizado e estruturado de coleta, descoberta e análise de informações sobre o ambiente de um determinado setor de atuação (TESTA, 2002 apud BACK, 2016).

Por sua vez,o CGEE (2006 apudBACK,2016) considera-o como um mecanismo que subsidia "[...] os processos de tomada de decisão e formulação de políticas públicas nos setores público e privado, por meio de abordagens baseadas em evidências e em percepções do futuro". 
Para Ibarrondo e Sánchez (2001), o observatório tem como função observar, analisar e difundir as informações estratégicas para auxiliar nas tomadas de decisão. Por sua vez, para Nascimento (2007), os observatórios, para atender seus objetivos, devem:

a) identificar conhecimentos explícitos disponíveis e do conhecimento tácito de especialistas;

b) articular organizações e indivíduos com participação colaborativa;

c) construir de visões de curto, médio e longo prazos sobre temas no quais a Ciência, a Tecnologia e a Inovação são aspectos centrais;

d) desenvolver processos de observação, monitoramento, antecipação e acompanhamento do desenvolvimento científico e tecnológico em áreas do conhecimento dos setores da economia e da sociedade de forma contínua ou discreta para atender necessidades específicas; e

e) estimular a capacidade das empresas em adotarem novas tecnologias por meio da identificação e disseminação de "boas práticas”empresariais, em nível nacional e internacional.

\subsubsection{Tipos}

No que tange aos tipos de observatório, David e Foray (2003) afirmam que estes podem ser classificados em função de algumas características, tais como: da geração e da internalização de novas competências e habilidades para a integração de ambientes de trabalhos dinâmicos; do acesso tanto à informação como ao conhecimento; do desenvolvimento diferenciado do conhecimento científico, tecnológico e organizacional; do conhecimento de diferentes setores de atividades; da abordagem de direitos de propriedade intelectual e a apropriação do conhecimento, que, em geral, são oriundos tanto das universidades e instituições de ensino e pesquisa, das empresas e indústrias, bem como do próprio governo.

Ratificando esta ideia, Gusmão (2005) e De La Vega (2007) detalharam o que denominaram de tipologias de observatórios, destacando suas relações com governos, universidades e indústrias. Segundo os autores, os observatórios podem estruturar-se das seguintes formas:

a) modelo tipo consórcio: apresenta estruturas relativamente autônomas, de caráter essencialmente público, reunindo agências, ministérios, instituições de pesquisa e/ou representantes do setor produtivo.Possuem maior grau de flexibilidade e de articulação entre os diversos atores do sistema nacional de C\&T e maior autonomia na adoção de um programa de trabalho próprio;

b) sob tutela do Ministério de C\&T: estruturas com caráter governamental, ligadas diretamente às decisões políticas e à formulação de estratégias nacionais para o setor. Possuem menor grau de autonomia operacional e financeira e maior vulnerabilidade face a eventuais entraves burocráticos;

c) de natureza acadêmica: núcleos nascidos no interior das universidades, a partir de trabalhos de cunho teórico-metodológico, desenvolvidos em parcerias com grupos de pesquisa das universidades de tutela. Possuem maior liberdade programática; 
d) núcleos de informação e documentação de agências governamentais do setor:são estruturas pequenas, especializadas, que operam em organismos já consolidados em atividades de gestão da informação científica (conselhos nacionais de C\&T, institutos nacionais de informação e documentação científica, institutos de pesquisa tecnológica etc.); e) redes ou estruturas de cooperação multilateral: reúne agências, conselhos de C\&T e/ou institutos de estatística de diferentes países com vistas à concepção, à definição e ao uso de indicadores regionais de C\&T.

\section{PROCEDIMENTOS METODOLÓGICOS}

Este artigo se utiliza da abordagem qualitativa, com busca sistemática nas bases de dados SCOPUS, SCIELO e em sites de observatórios acessíveis por intermédio de mecanismos de busca (Google e Bing). Os constructos de pesquisa adotados foram: conhecimento, inovação e observatório. O período de busca compreendeu os meses de novembro e dezembro de 2018. Não foram incluídos observatórios de inovação em fase de implantação, nem observatórios que não tivessem em seu propósito principal fomentar a inovação, independente da área.

\section{RESULTADOS E DISCUSSÕES}

\subsection{Principais observatórios}

\subsubsection{No Brasil}

Segundo levantamento realizado por Tavares et al. (2014), apesar da variedade de nomenclaturas, objetivos e públicos, nota-se que praticamente todos os observatórios visam fomentar a tomada de decisões. O número de observatórios ainda é pequeno no Brasil, não ultrapassando duas dezenas. A lista de alguns observatórios existentes está descrita a seguir.

a) OBSERVATÓRIO DA INOVAÇÃO E COMPETITIVIDADE (OIC) UNIVERSIDADE DE SÃO PAULO: O OIC está ligado ao Núcleo de Apoio à Pesquisa da Universidade de São Paulo (USP) e se propõe a elaborar pesquisas, estudos e análises sobre inovação e sociedade do conhecimento, de modo a contribuir com geração de conhecimento e, através dela, propiciar melhor discussão de políticas públicas e de estratégias empresariais pró-inovação (USP, 2013).

b) OBSERVATÓRIODEINOVAÇÃOSEBRAE: O Observatório de Inovação do SEBRAE objetiva servir como ferramenta para a obtenção de informações atualizadas sobre o potencial inovador das micro e pequenas empresas (MPE) das atividades indústrias e dos serviços considerando número de empresas, emprego (pessoas ocupadas) e a distribuição territorial das MPE. Suas ações consistem na identificação das micro e pequenas empresas inovadoras ou potenciais 
inovadoras (MPEs-PI), além de oferecer informações (tabelas e mapas) sobre empresas com até 99 pessoas ocupadas, ativas e registradas no Cadastro Nacional da Pessoa Jurídica (CNPJ), e com atividade principal na indústria e nos serviços disponíveis (SEBRAE, 2013).

c) OBSERVATÓRIO DE INOVAÇÃO DO TURISMO: Vinculado ao Núcleo de Estudos Avançados em Turismo e Hotelaria (NEATH), da Fundação Getúlio Vargas-RJ, o observatório visa desenvolver o pensamento estratégico para atividade turística, pensar estratégias para o setor e propor soluções que contribuam para o desenvolvimento do turismo no país. Atua durante a fase inicial deste projeto, buscava-se identificar as tendências do turismo internacional, avaliar os casos de sucesso aproximando-os da realidade brasileira, processá-los fomentando a discussão acadêmica e difundir o conhecimento gerado a partir desses trabalhos. Desde 2004, estuda os principais gargalos e entraves ao desenvolvimento do turismo. Em 2005, novas áreas foram propostas (OBSERVATÓRIO DE INOVAÇÃO DO TURISMO, 2013).

d) OBSERVATÓRIO IPEA DE GESTÃO DO CONHECIMENTO E INOVAÇÃO NA ADMINISTRAÇÃO PÚBLICA (OIGC): É um espaço virtual para disponibilizar informações e conhecimentos sobre como implantar a Gestão do Conhecimento na administração pública para inovar processos, produtos e serviços e para produzir resultados em benefício do cidadão. Entre os objetivos está: atuar como catalizador de análises e projetos de pesquisa sobre a GC e inovação na administração pública, a partir da disponibilização de base de dados consolidadas sobre temas relevantes para a implantação de boas práticas no serviço público, além de fornecer boletins com a análise dos principais fatos e tendências nesse segmento (IPEA, 2019).

e) OBSERVATÓRIO DE INFORMAÇÃOETECNOLOGIA:O observatório é vinculado ao setor de informação da Fundação CERTI e visa ao desenvolvimento e ao estímulo para a geração de novos empreendimentos, inovação e a produção de novos conhecimentos. Sua função é o monitoramento de informações que possibilitem a geração de novas ideias e oportunidades. Seu objetivo é criar um ambiente de comunicação e compartilhamento de informações. Tem como principais ações: reunir, organizar e oferecer referências e oportunidades que potencializem sua utilização, conhecimento e uso (OBSERVATÓRIO DE INFORMAÇÃO ETECNOLOGIA, 2013).

f) CENTRO INTERNACIONAL DE INOVAÇÃO (C2I): O centro é ligado ao Sistema Federação das Indústrias do Estado do Paraná (FIEP) e tem o objetivo de ser uma concessionária de inovação e articula produtos e serviços de inovação do SESI, SENAI, IEL e parceiros do Sistema FIEP. O C2I também oferece educação voltada para a gestão das empresas que inovam ou querem inovar por meio da Unindus, a Universidade da Indústria. O C2I age em oito principais domínios: criatividade, tecnologia, empreendedorismo, capital, design, inovação em negócios, sustentabilidade 
e gestão de conhecimento - e oferece produtos e serviços nessas áreas. Atua num programa para todas as indústrias nas principais áreas de Conhecimento e Inovação, Educação e Formação, Inteligência Consultiva e Sensibilização eMobilização.Além disso, articula ações ligadas à inovação ao lado de parceiros como UFPR, Instituto de Matemática Industrial, Paraná Metrologia, Junior Chamber Internacional Brasil, Anpei, Centro de Desing Paraná, Endeavor, entre outros (C2I, 2013).

g) ObservatóriodeInovaçãodaFIOCRUZ: Após um processo de reformulação iniciado em fevereiro de 2018, o Portal do Observatório em Ciência, Tecnologia e Inovação em Saúde da Fiocruz entrou novamente no ar no final de 2018 . O Observatório nasceu da necessidade de diagnosticar as atividades da Fiocruz nos campos da pesquisa e do ensino para além da lógica convencional, produtivista, que valoriza, sobretudo, a quantidade de publicações científicas. No Portal do Observatório, as informações sobre a Fiocruz podem ser acessadas no formato de indicadores interativos, de estudos quanti-qualitativos, bem como em referências disponíveis para consulta (FIOCRUZ, 2018).

h) Observatório de Ciência, Tecnologia e Inovação (CT\&I): Criado pela Secretaria de Estado de Ciência, Tecnologia e Ensino Superior (Sectes-MG) e coordenado pela Fundação João Pinheiro a partir de 2011, o Observatório corresponde a um depositário de bancos de dados que trata e analisa indicadores e informações. Seu objetivo geral é dotar o usuário de um sistema de bases de dados relevantes na área de CT\&I para subsidiar a formulação de políticas públicas voltadas para o desenvolvimento científico e tecnológico. O Observatório reúne e disponibiliza dados e indicadores relativos aos gastos dos governos estaduais em ciência e tecnologia e na atividade de pesquisa e desenvolvimento (P\&D), informações sobre o quantitativo de pesquisadores por área de conhecimento e indicadores de inovação tecnológica, entre outros (FJP, 2017).

Avaliando os Observatórios de Inovação encontrados no Brasil, percebe-se que ainda são poucos em quantidade e representatividade. A maioria está ligada a alguma instituição pública, seja universidade, fundação ou serviço social (Sistema "S"). Praticamente todos eles visam atender a um segmento específico, fornecendo informações para subsidiar decisões por parte dos associados, pesquisadores ou empresários no que tange à inovação em suas áreas de atuação. Quase todos surgiram na última década, demonstrando que esta ferramenta é bastante recente no país. Dentre as ferramentas mais utilizadas para compartilhamento de informações, estão os boletins e os indicadores.

Outra constatação importante é a existência de alguns observatórios de inovação social, tais como o Observatório de Inovação Social de Florianópolis (OIS-UDESC) e o Observatório de Inovação para Cidades Sustentáveis (em implantação pelo Ministério da Ciência, Tecnologia,Inovações e Comunicações). Estes observatórios conectam um conjunto abrangente de desafios urbanos nos temas de mobilidade, energia, água, resíduos sólidos, ambiente construído, soluções baseadas na natureza, inovação, visão e planejamento. 


\subsubsection{No mundo}

Back (2016), por sua vez, realizou importante mapeamento dos observatórios em diferentes lugares do planeta. Apesar de a autora citar a grande dificuldade do trabalho, haja vista a falta de um padrão de nomenclatura, pois alguns observatórios apresentam nomes diferentes mesmo tendo objetivos semelhantes, ela fez uso de três fontes principais, as quais colecionaram referências nos mais diferentes países: Nascimento (2007), Trzeciak (2009) e AdmNet (2012). A partir dessas, somou-se um levantamento realizado por meio da internet $\mathrm{e}$ iniciado no ano de 2012, buscando por observatórios que focassem na oferta de produtos de informação estratégica e de inteligência, para governos, universidades e empresas, com o objetivo de subsidiar decisões e promover a inovação. Ao todo, considerando o exposto, foram identificados 51 observatórios, distribuídos em 19 países, conforme se apresenta na Figura 2.

Figura 2: Observatórios mapeados por país

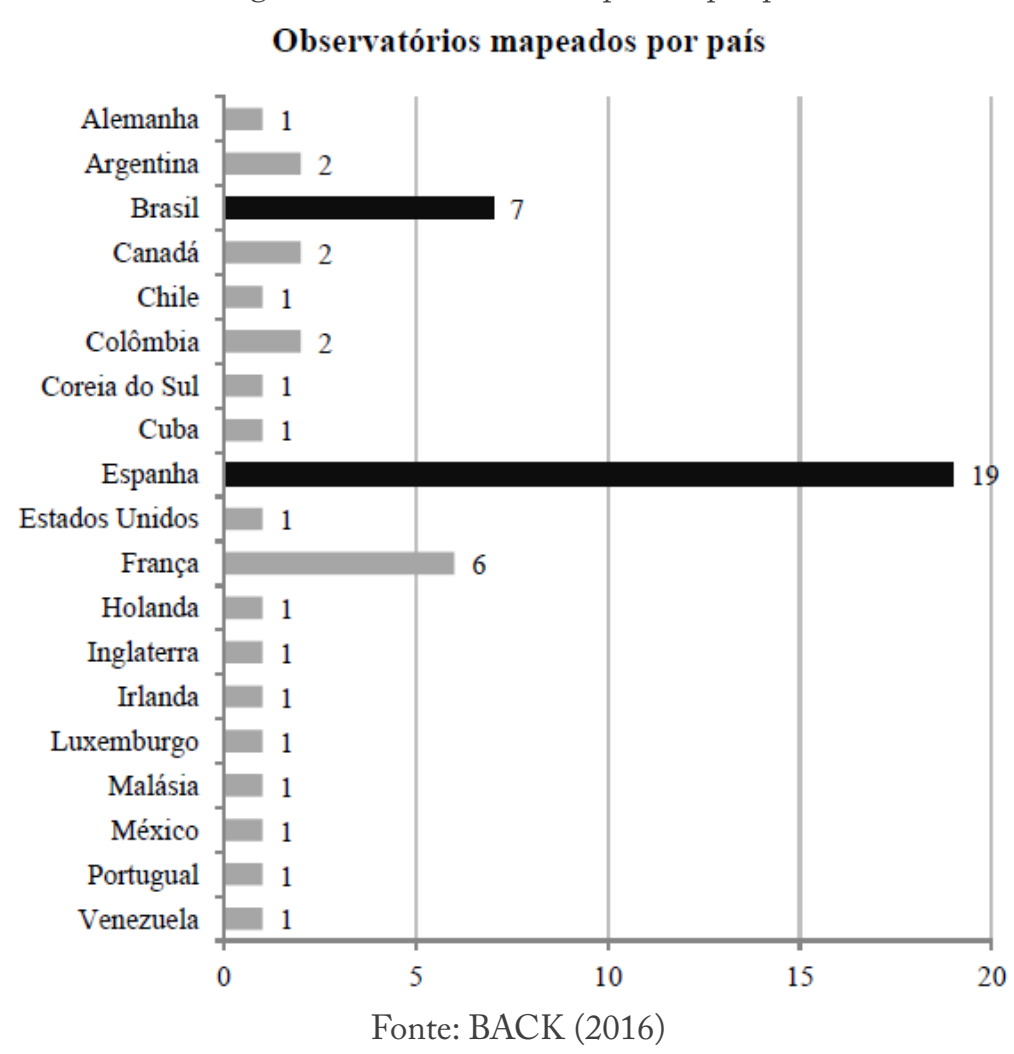

A realidade dos Observatórios no mundo não é muito diferente da realidade brasileira. Aliás, o Brasil tem figurado com número razoável, liderando em quantidade na América.Acontece que, conforme bem destacou Back (2016), as nomenclaturas muitas vezes podem prejudicar este levantamento, uma vez que entidades com outros nomes podem executar as atividades típicas de um observatório. Por exemplo,é sabido que países como os EUA investem forte em pesquisas na área de inovação, logo, as atividades de um Observatório podem estar contempladas em institutos, núcleos, centros de pesquisas e outras denominações que dificultam a rastreabilidade pela terminologia “Observatory”. Ainda assim, numa simples busca no site da Google 
com a expressão "Innovation Observatory", encontram-se mais de 500 mil ocorrências, sendo a grande maioria na América e Europa. Dentre os principais, aqueles com maior número de consultas e referências, destacam-se: Business Innovation Observatory (Europa),Technology Transfer and Innovation Observatory (Argentina) e Research and Innovation Observatory (Europa).

Os observatórios voltados à inovação social também apresentam crescimento importante nos últimos anos, especialmente aqueles voltados à resolução de problemas sociais, patrimônio histórico e de sustentabilidade nas grandes cidades, tais como: Eco Innovation Observatory (Europa) e o HESIOD (Inglaterra). O HESIOD - Observatório de Patrimônio Histórico e Social da Inglaterra é uma plataforma que visa identificar, analisar, dar visibilidade e disseminar experiências socialmente inovadoras no campo do patrimônio cultural: museus, projetos colaborativos, laboratórios de inovação, centros comunitários, espaços de trabalho compartilhados, cocriação, processos de coprodução, crowdsourcing e crowdfunding, etc. O Hesiod faz parte de um projeto de pesquisa sem fins lucrativos desenvolvido nas universidades de Oxford e UCL.

\subsection{Observatório de} Inovação como ferramenta de subsídio à inteligência nas organizações

Conforme o referencial teórico, é possível analisar os observatórios a partir dos conceitos de gestão do conhecimento e inovação. Afinal, o observatório, por meio de suas atividades e processos, busca informação estratégica para apoiar o processo de inovação nas organizações e, para isto, uma forte base de conhecimento é fundamental constitui-se em sua principal matéria-prima (BACK, 2016).

Um exemplo prático do anteriormente expostoé o Observatório de Inovação e Competitividade da USP - OIC. Os trabalhos desenvolvidos no OIC buscam enxergar além dos muros da academia, para que o conhecimento sirva também como fio guia para a implementação de políticas públicas e estratégias de incentivo à inovação. A instituição acredita que os debates desenvolvidos darão suporte para a melhoria da competitividade de vários setores da economia brasileira, derrubando pressupostos e ideias que foram durante muito tempo um entrave à cooperação entre a academia e os setores público e privado.

Assim sendo, destaca-se como o mais relevante resultado na elaboração de um modelo de observatório de inovação a constituição de em um mecanismo de inteligência voltado ao apoio do processo de inovação nas diferentes organizações. Aliás, ao se analisar os observatórios, verifica-se que:

1. o tipo de atividade que desenvolvem é baseado em conhecimentos, tanto do ponto de vista das entradas como das saídas dos seus processos;

2. o papel do conhecimento na criação de valor, que se configura como elemento primordial, já que se propõe a apoiar a criação de conhecimento organizacional voltado para a inovação; 
3. a dependência da atividade intelectual dos colaboradores, estes altamente qualificados, desenvolvendo desde as atividades de coleta e categorização de informações, passando pelas análises de especialistas, até a configuração de um sistema de informação que permita a disseminação desses conhecimentos;

4. o tipo de produto ou serviço oferecido, todos baseados em conhecimentos;

5. o mercado de atuação, que pode ser definido como o de organizações que buscam aprimorar seus processos de inovação; e

6. as práticas de gestão do conhecimento, que, assim como as de inteligência competitivas e prospecção,são próprias das desenvolvidas pelos observatórios (BACK, 2016).

Todas as fases do processo de inovação são suplementadas poralgum processo de inteligência, principalmente IC-Inteligência Competitiva, que oferece informação a cada uma delas, qualificando-as e apoiando no seu desenvolvimento, "[...] além de agregar valor ao resultado final-a inovação.”(TRZECIAK, 2009). Desta forma, informações e conhecimentos obtidos, entre outros, por meio dos processos de Inteligência (Competitiva, de Mercado, Estratégica etc.), tornam-se matéria-prima essencial ao processo de inovação (TIDD et al., 2008).

Ainda assim, é importante destacar que inovar não é tão simples e fácil, pois depende de diversas variáveis. Entre os principais obstáculos à inovação estão os custos, os riscos, a escassez de fontes de financiamento, a falta de acesso à informação sobre o mercado e tecnologias, os empecilhos burocráticos para fortalecer as cooperações e a falta de profissionais qualificados (BACK,2016).

\section{CONCLUSÃO}

A inovação é um processo que depende do nível de informação e conhecimento. Deste modo, pode-se considerar que há dependência dos elementos proporcionados pela informação na geração de novas estratégias de inovação. Diante disso, os observatórios podem ser considerados como centros articuladores, formuladores e disseminadores de conhecimento, tendo papel importantíssimo no processo de inovação.

As informações trazidas pelos Observatórios podem contribuir significativamente para a geração de valor, tornando possível enfrentar os variados e complexos desafios impostos às organizações, sempre buscando maior eficiência e eficácia nas ações executadas.
Embora se tenha observado a existência de diferenças significativas entre os diversos modelos de observatórios apresentados, tais como nomenclaturas, objetivos, escopo de abrangência, estrutura e público beneficiado, de forma geral, todos buscam reforçar o processo de tomada de decisão, subsidiando o uso da inteligência, especialmente a IC-Inteligência Competitiva.

Diante dos desafios postos, considerando que o processo de inovação se inicia na busca por insights e vai até a difusão das inovações desenvolvidas, ressalta-se que é de suma importância a consideração de dois aspectos: 
a) Avaliar até que pontos as informações disponibilizadas pelos principais observatórios de inovação ajudam no processo de inovação empresarial. Ou seja, até que ponto as informações são realmente úteis.

b) Observar o fenômeno das consequências da adoção de práticas inovadoras. Isso representará um passo significativo para a compreensão do desenvolvimento de ações inovadoras dentro do processo de inovação.
Por fim, aconselha-se o aprofundamento, para a melhor compreensão, de como atuam os observatórios de inovação, especialmente a partir da identificação de experiências práticas.

Assim sendo, pesquisas futuras devem examinar fatores adicionais especialmente relacionados aos impactos da gestão das informações e do conhecimento no processo de tomada de decisão no processo de inovação. 


\section{INNOVATION \\ OBSERVATORY AS AN \\ INTELLIGENCE TOOL IN ORGANIZATIONS}

\begin{abstract}
With increasingly dynamic social, political and economic scenarios, important transformations have been taking place in our society; leading companies to face the most varied and complex problems. Thus, innovative ideas are fundamental to respond to the demands of the market and to solve the main organizational problems. The innovation process does not occur in the company in isolation, it is interactive and of a social nature, counting on the contribution of several economic and social agents, holders of different types of information and knowledge, inside and outside the company. The Innovation Observatory is a space to show, articulate, support and accompany the actors that make up the Innovation Ecosystemnetwork in organizations, thus acting as an intelligence mechanism that supports the process of business innovation. In this way, this article aims to present the main concepts and models of Innovation Observatories existing in theliterature, as well as to highlight its importance as a subsidiary element for the decision making process when the subject involves innovation. The methodology used was the bibliographical research, which was based on secondary sources of data (SCOPUS and SCIELO base), correlating the themes knowledge management, innovation, intelligence and observatories. It is concluded that there are several initiatives in Brazil and in the world, however, it is noted the lack of a standard regarding the objectives, scope of scope and results obtained. In other words, the biggest challenge is to make information increasingly organized and disseminated by Observatories.
\end{abstract}

KEYWORDS: Knowledge management, innovation, Intelligence, observatory.

\section{REFERÊNCIAS}

ADAMS, G. L.; LAMONT, B. T. Knowledge management systems and developing sustainable competitive advantage. Journal of Knowledge Management, Aubum, v. 7, n. 2, p. 142-154, Aug. 2003.

BACK, S. Modelo de Observatório para apoio ao processo de inovação nas organizações: aplicação para as indústrias brasileiras de Bens de Capital. 2016. 376 f. Tese (Doutorado em Ciência e Engenharia de Materiais) - Universidade Federal de Santa Catarina, Florianópolis, 2016.

BARBIERI, J. C.; ÁLVARES, A. C. T. O retorno dos sistemas de sugestão: abordagens, objetivos e um estudo de caso. Cadernos EBAPE. BR, São Paulo, v. 3, Nesp, p. 01-17, mar. 2005.

CASTRO, D. Gestão da Inovação no setor público: um estudo de caso na regional Centro Sul de Minas dos Correios. Dissertação de Mestrado. Universidade Federal de Lavras. 2016. Lavras-MG.

CASTRO, Alexandre Camargo; JANNUZZI, Celeste Aída Sirotheau Corrêa; MATTOS, Fernando Augusto Mansor de. Produção e disseminação de informação tecnológica: atuação da Inova - Agência de Inovação da UNICAMP. Transinformação, Campinas, v. 19, n. 3, p. 265277, set./dez. 2007. Disponível em: Acesso em: 31 jan. 2019.

DAMANPOUR, F.; WALKER, R. M.; AVELLANEDA, C. N. Combinative effects of innovation types and organizational performance: a longitudinal study of service organizations. Journal of Management Studies, Newark, v. 46, n. 4, p. 650-675, June 2009.

FERRARESI, A. A. et al. Knowledge management and strategic orientation: leveraging innovativeness and performance. Journal of Knowledge

Management, Curitiba, v. 16, n. 5, p. 688-701, jun. 2012.

GUNDLING, E.; PORRAS, J. I. The 3M Way to innovation: balancing people and profit. Revista de Administração de Empresa, São Paulo, v. 41, n. 3, p. 219-229, jul./set. 2000. 
FIEDLER, L. Gestão de ideias para inovação contínua. Revista de Administração

Contemporânea, Curitiba, v. 15, n. 1, p. 173-175, jan./fev. 2011.

FIOCRUZ. Fundação Oswaldo Cruz. Portal do Observatório em CT\&I da Fiocruz é relançado com nova proposta. Disponível em: http://observatorio.fiocruz.br/noticias/portal-doobservatorio-em-cti-da-fiocruz-e-relancado-comnova-proposta. Acesso em: 06 mar. 2019.

FJP. Fundação João Pinheiro. Observatório de Ciência, Tecnologia e Inovação (CT\&I). Disponível em: http://www.fjp.mg.gov.br/index. $\mathrm{php} /$ produtos-e-servicos1/2730-observatorio-deciencia-tecnologia-e-inovacao-ct-i. Acesso em: 06 mar. 2019.

FLYNN, M. et al. Idea management for organisational innovation. International Journal of Innovation Management, Galway, v. 7, n. 4, p. 417- 442, Sept. 2003.

KOCK, A.; HEISING, W.; GEMUENDEN, H. $\mathrm{G}$. How ideation portfolio management influences front-end success. Journal of Product Innovation Management, Darmstadt, v. 32, n. 4, p. 539-555, July 2015.

LASTRES, H. M. M.; Arroio, LEMOS A. C. Políticas de apoio a pequenas empresas: do leito de Procusto à promoção de sistemas produtivos locais. In Lastres, H. M. M. et al., Pequena Empresa: cooperação e desenvolvimento local. Rio de Janeiro: Relume Dumará, 2003.

LASTRES, Helena M. M.; CASSIOLATO, José E. Glossário de Arranjos e Sistemas Produtivos e Inovativos Locais. REDESIST, 2003.

LIU, J. J.; CHEN, J. Y.; TAO, Y. Innovation performance in new product development teams in China's technology ventures: the role of behavioral integration dimensions and collective efficacy. Journal of Product Innovation Management, Zhejiang, v. 32, n. 1, p. 29-44, Jan. 2015.

MIGUEZ, Viviane Brandão. Uma abordagem de geração de ideias para o processo de inovação. Dissertação de Mestrado. Programa de Pós-Graduação em Engenharia e Gestão do Conhecimento. Florianópolis: Universidade Federal de Santa Catarina, 2017.
MOUSTAGHFIR, K. et al. Knowledge, learning, and innovation: research and perspectives. Journal of Knowledge Management, Ifrane, v. 17, n. 4, p. 495510, June 2013.

NONAKA, Ikujiro; TAKEUCHI, Hirotaka. Criação de conhecimento na empresa. Rio de Janeiro: Campus, 1997.

OBISF. O que é o Observatório de Inovação Social. Disponível em: http://www.observafloripa.com.br/ is-page//whatIs. Acesso em: 05 mar. 2019.

OECD - Organização para a cooperação econômica e desenvolvimento. Manual de Oslo: Proposta e Diretrizes para Coleta e Interpretação de dados sobre Inovação Tecnológica. Brasília: Finep, 2005.

PPGEGC - Programa de Pós-Graduação em Engenharia e Gestão do Conhecimento. Áreas de Concentração. 2018. Disponível em: http://www. egc.ufsc.br/pos-graduacao/programa/areas-deconcentracao/. Acesso em: 15 out. 2018.

RONGAGLIO, J. D. Observatório de inovação tecnológica da Fundação CERTI. TCC (graduação em Biblioteconomia) - Universidade Federal de Santa Catarina, Florianópolis. Disponível em: https:// repositorio.ufsc.br/handle/123456789/119540. Acesso em: 15 jan. 2019.

RUIZ-JIMÉNEZ, J. M.; DEL MAR FUENTESFUENTES, M. Knowledge combination, innovation, organizational performance in technology firms.

Industrial Management \& Data Systems, Granada, v. 113, n. 4, p. 523-540, Oct. 2013.

ŠKERLAVAJ, M.; ČERNE, M.; DYSVIK, A. I get by with a little help from my supervisor: Creativeidea generation, idea implementation, and perceived supervisor support. The Leadership Quarterly, Ljubljana, v. 25, n. 5, p. 987- 1000, May 2014.

SOUZA, J. A.; FREIRE, P. de S. Gestão estratégica de TI: notas de aula. Programa de Pós-Graduação em Engenharia e Gestão do Conhecimento. Florianópolis: UFSC, 2018.

SVEIBY, K. E. A nova riqueza das organizações. Rio de Janeiro: CAMPUS, 1998 


\section{SOBRE OS AUTORES}

TESTA, P. Indicadores científicos y tecnológicos en Venezuela: de las encuestas de potencial al observatorio de ciencia, tecnología e innovación. Cadernos del Cendes, Caracas, v. 19, n. 51, p. 43-64, set., 2002. Disponível em: http:// www.scielo.org.ve/scielo.php?pid=s1012$25082002000300004 \&$ script=sci_arttext. Acesso em: 19 jan. 2019.

TAVARES, L. E. S.; PEREIRA, A. C.; MOREIRA, I. P. A estruturação de um centro de inovação: $\mathrm{O}$ caso de estudo de um centro de inovação com um observatório para apoiar o setor de tecnologia de informação. In: XXIII Seminário Nacional de Parques Tecnológicas e Incubadoras de Empresas. / IASP 30th World Conference of Science Parks and Areas of Innovation, 2013, 2013, Recife. Anais dos Trabalhos Selecionados pela ANPROTEC., 2013. v. Unico.

TIDD, J.; BESSANT, J.; PAVITT, K. Gestão da Inovação. São Paulo: Bookman, 3. ed. 2008

TRZECIAK, D. S. Modelo de observatório para arranjos produtivos locais. 2009. $236 \mathrm{f}$. Tese (Doutorado em Engenharia de Produção) - Universidade Federal de Santa Catarina, Florianópolis, 2009.

VARVAKIS, G. DÁVILA, G. A. LEOCÁDIO, L. Inovação e Gerenciamento de Processos: uma análise baseada na Gestão do Conhecimento. Disponível em: http://www.ngs.ufsc.br/wpcontent/uploads/2010/06/2008_DataGramaZero_ Inovacao_GP.pdf. Acesso em: 19 jan. 2019.

YUSUF, S. From creativity to innovation.

Technology in Society, Washington, v. 31, n. 1, p. 1-8, Oct. 2009.

WALKER, R. M. Innovation type and diffusion: an empirical analysis of local government. Public Administration, Cardiff, v. 84, n. 2, p. 311-335, June 2006.

Data de recebimento: 06/05/2019

Data de aprovação: 27/08/2019

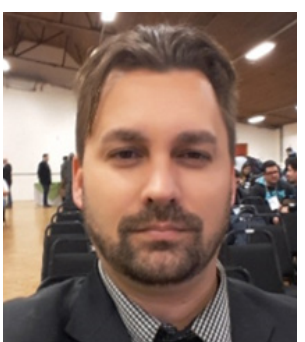

Thiago Zschornack

Gra d u a d o

e $\mathrm{m}$

Administração (Faculdade CNEC), Ciências Contábeis (UNISUL), Sistemas de Informação (UDESC), Gestão deTI(UNISUL) e Engenharia de Produção (UNICESUMAR).Possui 10 especializações completas, incluindo um MBA em Gestão Ambientale umMBA Executivo em GerenciamentodeProjetos (UGF). Mestre em Saúde e Meio Ambiente (UNIVILLE). Doutorando em Engenharia e Gestão do Conhecimento (UFSC). É professor universitário de graduação e pós-graduação na UNIVILLE, SENAC, UNISOCIESC e CENSUPEG. É conselheiro de Administração na Companhia Águas de Joinville, IVC e SENAC. É autor de três livros, dois cursos de especialização e é membro de diversos comitês ligados à área da qualidade e meio ambiente na cidade de Joinville. Pertence ao grupo de pesquisa IGTI-Inteligência, Gestão e Tecnologia para Inovação da UFSC (www.igit.ufsc.br).

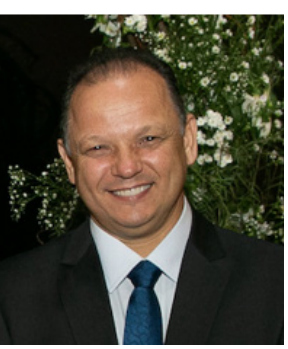

João Artur de Souza

Graduação em Matemática (Licenciatura) pela Universidade Federal de Santa Catarina (1989), mestrado em Matemática e Computação Científica pela Universidade Federal de Santa Catarina (1993), doutorado em Engenharia de Produção pela Universidade Federal de Santa Catarina (1999) e pós-doutorado pela Universidade Federal de Santa Catarina (2000). Trabalhou na Universidade Federal de Pelotas de 1993 à 2007 como professor na área de Matemática, atuando 
especialmente em Educação a Distância.Atualmente é Professor Associado da Universidade Federal de Santa Catarina do Departamento de Engenharia do Conhecimento. $\mathrm{Na}$ graduação tem trabalhado com disciplinas Inteligência Artificial e Lógica Matemática. Na pós-graduação atua no Programa de Pós-Graduação em Engenharia e Gestão do Conhecimento da UFSC na área de Inteligência e Gestão para Inovação. Pertence ao grupo de pesquisa IGTI-Inteligência, Gestão e Tecnologia para Inovação (www.igit.ufsc.br).

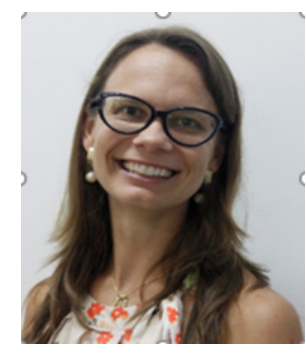

\section{Luana Siewert Pretto}

Engenheira Civil (UFSC) com MBA em gerenciamento de Projetos (FGV) e Mestrado em Análise Multicritério para tomada de decisão (UFSC). Doutoranda em Engenharia do Conhecimento e Presidente da Companhia Águas de Joinville.

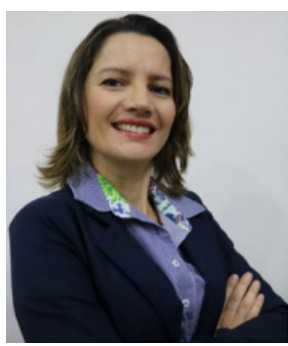

Adriana Falcão Loth

Doutoranda em Engenharia e Gestão do Conhecimento pela UFSC. Mestre em Gestão de Políticas Públicas. Especialista em diferentes áreas, como: Direito do Trabalho e Processos trabalhistas; Remuneração Estratégica; Treinamento e Desenvolvimento; Terapia de Família e casal; Infância e Violência Doméstica e Docência Superior na Área da Saúde. Graduada em Psicologia.Experiência profissional em diferentes áreas como: Psicologia Organizacional e Clínica, Saúde Pública, Educação Especial, Gestão de Pessoas,Liderança e Docência no Ensino Superior (graduação e pós-graduação).Atualmente trabalha na Companhia Águas de Joinville como Gerente de Gestão de Pessoas e como consultora de liderança. 